\title{
Non-obstetric diagnostic imaging in pregnancy
}

\author{
Ally Murji MD MPH, Rebecca Crosier HBSc, Pasteur Rasuli MD
}

\section{A fetal radiation dose of $\leq \mathbf{5 0}$ milli- Gray (mGy) is associated with negligi- ble risks}

Data from observational studies of pregnant survivors of nuclear disasters and experimental studies on animals have shown that, in the first two weeks after conception, the main risk is radiationinduced pregnancy termination, which can occur at a radiation dose of 100-200 mGy. From two weeks after conception until term, a minimum fetal radiation dose of $350-500 \mathrm{mGy}$ is required to cause adverse outcomes such as pregnancy loss, fetal malformations, growth restriction and developmental delay. ${ }^{1}$ Practice guidelines from the American Congress of Obstetricians and Gynecologists suggest a more conservative threshold of $50 \mathrm{mGy}$ or less during pregnancy to avoid adverse events. ${ }^{2}$

There is a weak association between antenatal ionizing radiation and childhood cancer

In case-control studies, ${ }^{4}$ in utero exposure to medical diagnostic radiation was associated with a discernable increase in relative risk of childhood leukemia. Given the low baseline rate of childhood cancer, the absolute increased risk from ionizing radiation is small. For example, an antenatal fetal dose of $20 \mathrm{mGy}$ translates to 0.8 additional childhood cancers per 100 babies. $^{4}$

CMAJ invites submissions to "Five things to know about ..." Submit manuscripts online at http://mc.manuscriptcentral .com/cmaj

\section{No single diagnostic procedure exceeds the threshold of 50 mGy}

Typical fetal radiation doses from common examinations are shown in Appendix 1 (available at www.cmaj.ca/lookup/suppl/doi:10.1503/cmaj.140901/-/DC1). ${ }^{3}$ For example, the fetal dose from chest radiographs is less than $0.01 \mathrm{mGy}$. More than 5000 such radiographs in pregnancy are required to exceed the 50-mGy threshold. By comparison, the fetal dose during a transatlantic flight is $0.01 \mathrm{mGy}$, and naturally occurring background radiation during pregnancy is $1 \mathrm{mGy}$.

\section{Guidelines consider magnetic reso- nance imaging (MRI) to be safe dur- ing the second and third trimesters}

The guideline on MRI imaging from the Society of Obstetricians and Gynaecologists of Canada is based on welldesigned cohort studies. ${ }^{5}$ Some animal studies have shown teratogenesis with MRI exposure in the first trimester, but no adverse effects in humans have been documented. ${ }^{5}$ Given the theoretical concern, MRI in the first trimester should be restricted to situations where other imaging modalities are inconclusive and maternal care depends on further imaging. ${ }^{5}$

\section{References}

1. Groen RS, Bae JY, Lim KJ. Fear of the unknown: ionizing radiation exposure during pregnancy. Am J Obstet Gynecol 2012;206:456-62.

2. ACOG Committee on Obstetric Practice. Guidelines for diagnostic imaging during pregnancy ACOG Committee opinion no. 299 September 2004 (replaces no. 158 September 1995). Obstet Gynecol 2004;104:647-51

3. McCollough $\mathrm{CH}$, Schueler BA, Atwell TD, et al Radiation exposure and pregnancy: When should we be concerned? Radiographics 2007;27:909-17.

4. Wakeford R. Childhood leukaemia following medical diagnostic exposure to ionizing radiation in utero or after birth. Radiat Prot Dosimetry 2008;132:166-74.

5. Patenaude Y, Pugash D, Lim K, et al; Society of Obstetricians and Gynaecologists of Canada. The use of magnetic resonance imaging in the obstetric patient. J Obstet Gynaecol Can 2014;36:349-63.

6. Chen MM, Coakley FV, Kaimal A, et al. Guidelines for computed tomography and magnetic resonance imaging use during pregnancy and lactation. Obstet Gynecol 2008;112:333-40.
Contrast agents are unlikely to cause fetal harm, but they should be used only when benefits of improved diagnostic sensitivity outweigh potential risks

Iodine-based contrast agents used in computed tomography cross the placenta and may theoretically affect fetal thyroid function. Similarly, gadolinium contrast medium for MRI crosses the placenta and enters the amniotic fluid, which results in prolonged fetal exposure. Animal studies are conflicting. Some have documented adverse effects with high-level exposure. No adverse outcomes have been documented in humans. ${ }^{6}$ Although no clinical sequelae from brief exposures to either agent in pregnant women have been reported, ${ }^{6}$ these compounds should be used judiciously.

Competing interests: Ally Murji has received speaker fees from Actavis, AbbVie, Bayer and Hologic. No other competing interests were declared.

Affiliations: Department of Obstetrics and Gynecology (Murji, Crosier), Mount Sinai Hospital, University of Toronto, Toronto, Ont.; Department of Diagnostic Imaging (Rasuli), University of Ottawa, Ottawa, Ont.

Correspondence to: Ally Murji, amurji@mtsinai. on.ca

CMAJ 2015. DOI:10.1503/cmaj.140901 